\title{
CoRoT data: final pipe-line for bright stars
}

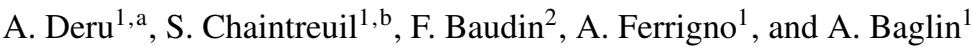 \\ 1 LESIA, Observatoire de Paris, CNRS (UMR 8109), Meudon France \\ 2 Institut d'Astrophysique Spatiale, Université Paris Sud, CNRS (UMR 8617), Orsay France
}

\begin{abstract}
The observations made by the CoRoT satellite were divided into short and long periods called runs; for a detailed description of the runs see [1]. The global processing pipeline is divided in two sub-pipelines: the first one is dedicated to the corrections that can be applied to simultaneous data, while the second one needs the whole duration of each run to be applied. We present here the final form of the entire processing pipeline for the bright stars channel, which was previously called the seismo channel.
\end{abstract}

\section{1 / Pipeline AN0 to AN1}

The first level of correction is applied to the raw data and is focused on the instrumental and environmental disturbances (see Fig. 1). At this level, only instantaneous corrections are performed, working on data taken at a given moment, and based on the calibrations made on board and on the ground [2].

First the electromagnetic interference is corrected using the patterns inferred from the calibrations, and the correction of offsets and background are performed in 2 steps. First they are mainly subtracted on board: for each bright star, a background window is placed in a dark region near the star and used to directly subtract the background level. And in a second time additional corrections are done on the ground. A proper dating is also applied to the data from the on board counter.

The movement of the barycenter of each star is recorded on board, and used on ground, to reconstruct as precisely as possible the line of sight of the satellite (LOS). With this LOS and the use of the surfaces of correction computed from the imagettes, the satellite jitter is estimated and corrected on

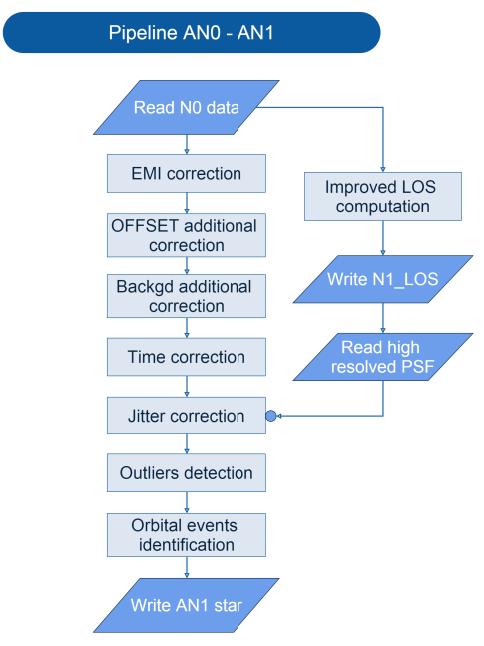

Fig. 1. Flowchart pipeline AN1 ground [3].

Then, outliers are detected using a moving median, and every difference greater than 5 times the standard deviation is marked as an outlier. To finish, the orbital events information is provided with the data, in particular:

- the SAA crossing: too many impacts to exploit the data,

- the entry and exit of the Earth's shadow: the variation of the temperature of the solar panels is sufficient to produce mechanical constraints that affect the pointing of the satellite.

\footnotetext{
a e-mail: alexis.deru@obspm.fr

b e-mail: sylviane.chaintreuil@obspm.fr
}

This is an Open Access article distributed under the terms of the Creative Commons Attribution License 4.0, which permits unrestricted use, distribution, and reproduction in any medium, provided the original work is properly cited. 


\section{2 / Pipeline AN1 to AN2}

Once N1 data are produced, a second level of correction is necessary to reach the scientific requirements of the mission. The successive corrections work on the whole duration of the signal [4].

The current AN2 data (v1.x to v3.x) are structured in 3 extensions, named extends; each extend corresponding

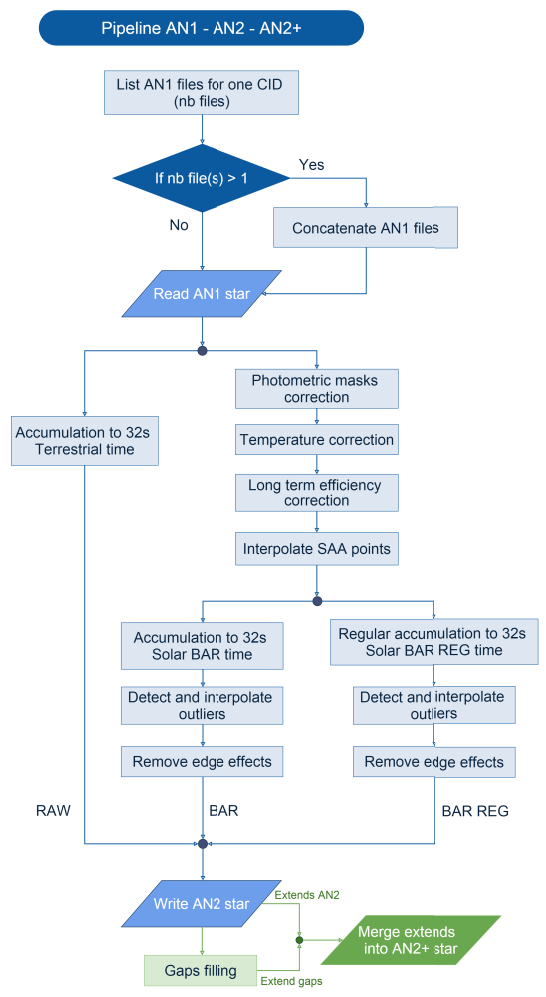

Fig. 2. Flowchart pipeline AN2 to a specific type of time correction. For the RAW extend, the Julian time is accumulated from a 1 second sampling period to a 32 second sampling period, and no further corrections are applied. For the 2 other extends, the first corrections are identical:

- Photometric mask correction: for each run there are 2 phases, a first short one with a rough mask to set the line of sight, and second one, with a larger mask, for the rest of the run. Using the 2 masks, the missing flux is computed directly from the imagettes and used to correct the first sequence.

- Temperature correction: during the mission, the CCDs temperature was controlled, and was corrected using several commands. Each command is compensated by measuring the height of the jump and its impact on the light curve.

- Long term efficiency: due to several causes (increase of the dark current, opacity of the focal box), the efficiency of the collection of photons decreases throughout the mission, causing a long-term trend. A correction factor, linked to the observed intensity of the star, is computed in the linear approximation and applied to the data.

At this point the time vector is converted to Heliocentric Julian Time and the 2 extends are processed separately. The first one is just accumulated on 32 second exposures (extend HEL), and the second one is regularly resampled before accumulated at 32s (extend HEL REG).

\section{3 / A final step: the AN2 legacy}

The final version of the data (v4.x), presented in Fig. 2, allows further steps of correction. First, the Terrestrial Time (TT) replaces the Universal Time (UT) and the Heliocentric Julian Date is converted to Solar Barycentric Time. In consequence, the extends HEL and HELREG are respectively renamed BAR and BARREG. Finally, a gap interpolation by Inpainting method [5] is implemented, and all the existing extends are merged in the final AN2 product.

\section{References}

1. CoRoT archive: IAS data center http://idoc-corot.ias.u-psud.fr/

2. M. Auvergne et al., A\&A 506, (2009) 411

3. F. De Oliveira Fialho et al., PASP 119, (2007) 337

4. R. Samadi and F. Fialho, ESA-SP 1306, (2006), 317

5. S. Pires, S. Mathur, R. A. García, J. Ballot, D. Stello, and K. Sato, A\&A 574, (2015) A18 